\title{
A PARTITION THEOREM FOR THE INFINITE SUBTREES OF A TREE \\ BY
}

KEITH R. MILLIKEN

\begin{abstract}
We prove a generalization for infinite trees of Silver's partition theorem. This theorem implies a version for trees of the Nash-Williams partition theorem.
\end{abstract}

1. Introduction. First we establish some notation. An ordinal will be identified with the set of smaller ordinals, and a cardinal will be an initial ordinal. For example, $4=\{0,1,2,3\}$; and $\omega=\boldsymbol{\aleph}_{0}$ is the set of all nonnegative integers as well as the cardinality of that set. If $X$ is a set, then $|X|$ is the cardinaltiy of $X$. If $\kappa$ is a cardinal, then $[X]^{\kappa}=\{Y \subseteq X:|Y|=\kappa\},[X]^{<\kappa}=\{Y \subseteq X:|Y|<\kappa\}$, and $[X]^{<\kappa}$ $=[X]^{<\kappa} \cup[X]^{\kappa}$.

In [2], Erdös and Rado made the following definition: a family of sets $\mathscr{F} \subseteq[\omega]^{\kappa_{0}}$ is Ramsey provided there exists $X \in[\omega]^{\alpha_{0}}$ with either $[X]^{\mu_{0}} \subseteq \mathcal{F}$ or $[X]^{\mu_{0}} \cap \mathscr{F}=\varnothing$. Erdös and Rado also proved that the axiom of choice implies that there exists $\mathcal{F} \subseteq[\omega]^{\kappa_{0}}$ that is not Ramsey.

However, $[\omega]^{\kappa_{0}}$ is naturally embedded in $2^{\omega}=\{f: f$ is a function from $\omega$ into 2$\}$, and so we can consider $[\omega]^{\kappa_{0}}$ with the induced topology, where $2^{\omega}$ has the Tychonoff product topology. In this topology, the work of Nash-Williams [8] and of Galvin and Prikry [3] shows that each Borel set is Ramsey. Silver [10] extended these results to show that every analytic set is Ramsey (see Corollary 1.12 below). And recently, Ellentuck [1] and others (see [5] and [11]) have found simpler proofs of Silver's result.

The primary result of this paper (Theorem 1.9 below) is a version for trees of Silver's theorem. This result for trees implies Silver's theorem. Also, just as Silver's theorem implies the Nash-Williams partition theorem (Theorem 3.1 below) and Ramsey's theorem, so our result implies a version for trees of the Nash-Williams theorem (Theorem 3.3 below) and a version for trees of Ramsey's theorem (Corollary 3.4 below). This last mentioned Ramsey's theorem for trees was originally proved in [6].

In order to work with trees, we need several definitions. These are listed together here for convenient reference.

Suppose $P=\langle P, \leqslant\rangle$ is a partially ordered set. (We use a single symbol both for a structure and for its underlying set.) If $p \in P$, we write

Received by the editors August 29, 1978 and, in revised form, January 21, 1980.

AMS (MOS) subject classifications (1970). Primary 04A20, 05C05, 06A10. 


$$
\begin{array}{ll}
\operatorname{Pred}(p, P)=\{q \in P: q \leqslant p\}, & \operatorname{Pred}^{*}(p, P)=\operatorname{Pred}(p, P)-\{p\} \\
\operatorname{Succ}(p, P)=\{q \in P: q \geqslant p\}, & \operatorname{Succ}^{*}(p, P)=\operatorname{Succ}(p, P)-\{p\} .
\end{array}
$$

We shall be primarily concerned with rooted trees of finite height or of height $\omega$, so the following definition of a tree will be used.

Definition 1.1. A tree $t=\langle T, \leqslant\rangle$ is a partially ordered set satisfying:

(1) $T$ has a unique least element, called the root of $T$ and $\operatorname{denoted} \operatorname{Root}(T)$, and

(2) for each $t \in T, \operatorname{Pred}(t, T)$ is a finite chain, i.e., $\operatorname{Pred}(t, T)$ is a finite, linearly ordered set in $\langle T, \leqslant\rangle$.

The elements of a tree $T$ will sometimes be called nodes. If $t \in T$, then the level of $t$ in $T$, denoted $\operatorname{Lev}(t, T)$, is the cardinality of $\operatorname{Pred}^{*}(t, T)$. If $n \in \omega, T(n)=$ $\{t \in T: \operatorname{Lev}(t, T)=n\}$, i.e., $T(n)$ is the set of nodes on the $n$th level of $T$. The height of $T$ is $\operatorname{Height}(T)=\sup \{|\operatorname{Pred}(t, T)|: t \in T\}$. For example, if $n \in \omega$ implies $T(n) \neq \varnothing$, then $T$ must have height $\omega$. A branch of $T$ is a maximal chain in $\langle T, \leqslant\rangle$. We call $T$ an $\alpha$-tree (where $\alpha \leqslant \omega$ ) provided each branch of $T$ has cardinality $\alpha$. Thus each $\alpha$-tree has height $\alpha$, but a tree with height $\alpha$ need not be an $\alpha$-tree.

If $s$ and $t$ are nodes of $T$, we say $s$ is an immediate successor of $t$ when $s$ is minimal in $\operatorname{Succ}^{*}(t, T)$, or equivalently, when $t=\max \left\{\operatorname{Pred}^{*}(s, T)\right\}$. We write IS $(t, T)$ for the collection of all immediate successors of $t$ in $T$.

If $\kappa$ is a cardinal (finite or infinite), and if $\alpha \leqslant \omega$, an $(\alpha, \kappa)$-tree is an $\alpha$-tree with each nonmaximal node having exactly $\kappa$ immediate successors. An $(\alpha,<\kappa)$-tree is an $\alpha$-tree with each nonmaximal node having fewer than $\kappa$ immediate successors, and an $(\alpha, \leqslant \kappa)$-tree is an $\alpha$-tree with each nonmaximal node having at most $\kappa$ immediate successors.

If $0 \leqslant \alpha \leqslant \beta \leqslant \omega$, we write $\operatorname{Incr}(\alpha, \beta)$ for the set of all strictly increasing functions from $\alpha$ into $\beta$.

Below is a formal definition of when a tree $S$ is strongly embedded in another tree $T$. Intuitively, for $S$ to be strongly embedded in $T, S$ must be a subset of $T$ with the induced partial order. $S$ must preserve the branching structure of $T$, i.e. given a (nonmaximal) node of $S$, if that node has $k$ immediate successors in $T$, then that node must have $k$ corresponding immediate successors in $S$. Also, $S$ must preserve the level structure of $T$, i.e. all nodes of $S$ on a common level (of $S$ ) must be from a common level in $T$.

Definition 1.2. Suppose $S$ is an $\alpha$-tree and $T$ is a $\beta$-tree with $0<\alpha<\beta<\omega$. $S$ is strongly embedded in $T$ provided the following hold.

(1) $S \subseteq T$, and the partial order on $S$ is induced from $T$.

(2) If $s \in S$ is nonmaximal in $S$ and $t \in \operatorname{IS}(s, T)$ then $\operatorname{Succ}(t, T) \cap \operatorname{IS}(s, S)$ is a singleton.

(3) There exists $f \in \operatorname{Incr}(\alpha, \beta)$ such that $S(n) \subseteq T(f(n))$ for each $n \in \alpha$.

The function $f$ in (3) is called the level assignment function for $S$ in $T$, and we write $f=\operatorname{LAF}(S, T)$.

Given $f \in \operatorname{Incr}(\alpha, \beta)$, we write $\operatorname{Str}_{f}(T)$ for the collection of all $\alpha$-trees strongly embedded in the $\beta$-tree $T$ that have $f$ as level assignment function in $T$. Also, we 
write

$$
\begin{aligned}
\operatorname{Str}^{\alpha}(T) & =\bigcup_{f \in \operatorname{Incr}(\alpha, \beta)} \operatorname{Str}_{f}(T), \\
\operatorname{Str}^{<\alpha}(T) & =\bigcup_{n \in \alpha} \operatorname{Str}^{n}(T), \\
\operatorname{Str}^{<\alpha}(T) & =\operatorname{Str}^{\alpha}(T) \cup \operatorname{Str}^{<\alpha}(T) .
\end{aligned}
$$

The proof we give of our main theorem involves consideration of finite sequences of trees. So we shall extend the above notation to finite sequences of trees. Suppose $d$ is a positive integer and $\left\langle T_{i}: i \in d\right\rangle$ is a sequence of $\beta$-trees for some $0 \leqslant \beta \leqslant \omega$. If $0 \leqslant \alpha \leqslant \beta$ and $f \in \operatorname{Incr}(\alpha, \beta)$, then we write

$$
\begin{aligned}
\operatorname{Str}_{f}\left(T_{i}: i \in d\right) & =\left\{\left\langle S_{i}: i \in d\right\rangle: S_{i} \in \operatorname{Str}_{f}\left(T_{i}\right) \text { for each } i \in d\right\} \\
& =\prod_{i \in d} \operatorname{Str}_{f}\left(T_{i}\right), \\
\operatorname{Str}^{\alpha}\left(T_{i}: i \in d\right) & =\bigcup_{f \in \operatorname{Incr}(\alpha, \beta)} \operatorname{Str}_{f}\left(T_{i}: i \in d\right), \\
\operatorname{Str}^{<\alpha}\left(T_{i}: i \in d\right) & =\bigcup_{n \in \alpha} \operatorname{Str}^{n}\left(T_{i}: i \in d\right) .
\end{aligned}
$$

$\operatorname{Str}^{<\alpha}\left(T_{i}: i \in d\right)$ is defined similarly.

It should be noted that if $S, R$ and $T$ are $\omega$-trees with $S \in \operatorname{Str}_{f}(T)$ and $R \in \operatorname{Str}_{g}(S)$, then $R \in \operatorname{Str}_{h}(T)$ where $h(n)=f(g(n))$ for each $n \in \omega$.

Definition 1.3. We write Id for the identity function on $\omega$, i.e., Id: $\omega \rightarrow \omega$ with $\operatorname{Id}(n)=n$ for each $n \in \omega$. Thus $\operatorname{Id} \mid n$, the restriction of Id to $n$, is the identity function on $n$.

Definition 1.4. Suppose $s$ is an $\alpha$-tree and $T$ is a $\beta$-tree for some $0<\alpha<\beta<$ $\omega$. Then $S$ is a strong initial segment of $T$ (denoted $S<^{*} T$ ) provided $S$ is the unique tree satisfying $S \in \operatorname{Str}_{\operatorname{Id|\alpha }}(T)$.

Definition 1.5. Suppose $T$ is an $\omega$-tree and $A \in \operatorname{Str}^{<\omega}(T)$. Then we shall write $\operatorname{Str}(A, T)=\left\{R \in \operatorname{Str}^{\omega}(T): A<{ }^{*} R\right\}$. So, in particular, $\operatorname{Str}(\phi, T)=\operatorname{Str}^{\omega}(T)$. Also, we shall write $\operatorname{Dmt}(A, T)$ for the maximal tree $\operatorname{str}(A, T)$ and call $\operatorname{Dmt}(A, T)$ the dominating tree of $A$ in $T$, i.e.,

$$
\operatorname{Dmt}(A, T)=A \cup\{\operatorname{Succ}(t, T): t \text { is a maximal node of } A\}
$$

where $\operatorname{Dmt}(A, T)$ has the partial order induced from $T$.

Definition 1.6. Suppose that $d$ is a positive integer, that $\left\langle T_{i}: i \in d\right\rangle$ is a sequence of $\omega$-trees, $n \in \omega, f \in \operatorname{Incr}(n, \omega)$, and that $A_{i} \in \operatorname{Str}_{f}\left(T_{i}\right)$ for each $i \in d$. We shall write

$$
\operatorname{Str}\left(A_{i}, T_{i}: i \in d\right)=\bigcup_{\substack{g \in \operatorname{Incr}(\omega, \omega) \\ g|n=\operatorname{Id}| n}}\left(\prod_{i \in d} \operatorname{Str}_{\boldsymbol{g}}\left(\operatorname{Dmt}\left(A_{i}, T_{i}\right)\right)\right) .
$$

Intuitively, $\operatorname{Str}\left(A_{i}, T_{i}: i \in d\right)$ consists of all sequences $\left\langle S_{i}: i \in d\right\rangle$ in $\operatorname{Str}^{\omega}\left(T_{i}: i \in d\right)$ that (for each $\left.i \in d\right)$ have $A_{i}$ being a strong initial segment of $S_{i}$.

Definition 1.7. Suppose $T$ is an $\omega$-tree and $R \subseteq \operatorname{Str}^{\omega}(T)$. We say $R$ is $T$-Ramsey provided there exits $T^{\prime} \in \operatorname{Str}^{\omega}(T)$ with either $\operatorname{Str}^{\omega}\left(T^{\prime}\right) \subseteq R$ or $\operatorname{Str}^{\omega}\left(T^{\prime}\right) \cap R=\varnothing$. 
Considering $\omega$ with its usual ordering as the trivial $(\omega, 1)$-tree, then $\omega$-Ramsey means just Ramsey in the traditional sense mentioned above.

Definition 1.8. Suppose $d$ is a positive integer and $\left\langle T_{i}: i \in d\right\rangle$ is a sequence of $\omega$-trees. We say that a set $R \subseteq \operatorname{Str}^{\omega}\left(T_{i}: i \in d\right)$ is completely $\left\langle T_{i}: i \in d\right\rangle$-Ramsey provided the following holds. If $\left\langle S_{i}: i \in d\right\rangle \in \operatorname{Str}^{\omega}\left(T_{i}: i \in d\right)$, and if $\left\langle A_{i}: i \in d\right\rangle$ $\in \operatorname{Str}^{<\omega}\left(S_{i}: i \in d\right)$, then there exists $\left\langle R_{i}: i \in d\right\rangle \in \operatorname{Str}\left(A_{i}, S_{i}: i \in d\right)$ such that either $\operatorname{Str}\left(A_{i}, R_{i}: i \in d\right) \subseteq R$ or $\operatorname{Str}\left(A_{i}, R_{i}: i \in d\right) \cap R=\varnothing$.

If $d=1$ so $\left\langle T_{i}: i \in d\right\rangle=\left\langle T_{0}\right\rangle$, we shall say $R$ is "completely $T_{0}$-Ramsey" instead of saying $R$ is "completely $\left\langle T_{0}\right\rangle$-Ramsey." So $R$ is completely $T$-Ramsey means that for each $S \in \operatorname{Str}^{\omega}(T)$ and each $A \in \operatorname{Str}^{<\omega}(S)$, there exists $S^{\prime} \in$ $\operatorname{Str}(A, S)$ with either $\operatorname{Str}\left(A, S^{\prime}\right) \subseteq R$ or $\operatorname{Str}\left(A, S^{\prime}\right) \cap R=\varnothing$. Clearly, if $R$ is complete $T$-Ramsey, then $R$ is $T$-Ramsey.

Given a sequence of $\omega$-trees $\left\langle T_{i}: i \in d\right\rangle$ where $d$ is a positive integer, we shall define a topology on $\operatorname{Str}^{\omega}\left(T_{i}: i \in d\right)$ by taking $\left\{\operatorname{Str}\left(A_{i}, S_{i}: i \in d\right):\left\langle S_{i}: i \in d\right\rangle \in\right.$ $\operatorname{Str}^{\omega}\left(T_{i}: i \in d\right)$ and $\left.\left\langle A_{i}: i \in d\right\rangle \in \operatorname{Str}^{<\omega}\left(S_{i}: i \in d\right)\right\}$ as a basis. This topology will be called the tree topology on $\operatorname{Str}^{\omega}\left(T_{i}: i \in d\right)$. If $t$ is a single $\omega$-tree, then the tree topology on $\operatorname{Str}^{\omega}(T)$ has $\left\{\operatorname{Str}(A, S): S \in \operatorname{Str}^{\omega}(T)\right.$ and $\left.A \in \operatorname{Str}^{<\omega}(S)\right\}$ as a basis.

For completeness, we also define the analytic sets in a topology. Suppose $\tau=\langle X, \mathbf{G}\rangle$ is a topological space, i.e., $X$ is a set and $\mathbf{G}$ is the family of open subsets $X$. Write $\mathrm{F}$ for the family of closed subsets of $X$. Suppose $T$ is an arbitrary $\left(\omega, \aleph_{0}\right)$-tree, and $\mathbf{B}$ is the set of all branches of $T$. Then $A \subseteq X$ is analytic in $\tau$ if there exists a function $f: T \rightarrow$ F such that

$$
A=\bigcup_{B \in \mathbf{B}}\left(\bigcap_{t \in B} f(t)\right) .
$$

It is well known that every Borel set is analytic.

Using these definitions, we can state our main theorem.

TheOREM 1.9. Suppose $T$ is an $\left(\omega,<\aleph_{0}\right)$-tree and $R \subseteq \operatorname{Str}^{\omega}(T)$ is an analytic set in the tree topology on $\operatorname{Str}^{\omega}(T)$. Then $R$ is completely $T$-Ramsey; hence $R$ is $T$-Ramsey.

First let us see how Theorem 1.9 implies Silver's partition theorem. If $A$ and $B$ are subset of $\omega$, we write $A<B$ to mean: for each $a \in A$ and $b \in B$, we have $a<b$.

Definition 1.10. If $A \in[\omega]^{<\kappa_{0}}$ and $X \subseteq \omega$, then we say $A$ is an initial segment of $X$ and write $A \ll X$ provided there exists $Y \subseteq \omega$ with $A<Y$ and $A \cup Y=X$.

If we consider $[\omega]^{\kappa_{0}}$ to be embedded in $2^{\omega}$ has the Tychonoff product topology, then we shall call the induced topology on $[\omega]^{\alpha_{0}}$ the classical topology. If for each $A \in[\omega]^{<*_{0}}$ we write

$$
I_{A}=\left\{Y \in[\omega]^{\aleph_{0}}: A \ll Y\right\}
$$

then $\left\{I_{A}: A \in[\omega]^{<\kappa_{0}}\right\}$ is a basis for the classical topology on $[\omega]^{\mu_{0}}$.

If we instead consider $\omega$ with the usual ordering to be the trivial $(\omega, 1)$-tree, then the tree topology on $\operatorname{Str}^{\omega}(\omega)=[\omega]^{\mu_{0}}$ is finer (has more open sets) than the classical topology. A typical basic open set for the tree topology on $\operatorname{Str}^{\omega}(\omega)=[\omega]^{\mu_{0}}$ is of the 
form

$$
J_{A, X}=\left\{Y \in[X]^{\aleph_{0}}: A \ll Y\right\}
$$

where $X \in[\omega]^{\kappa_{0}}$ and $A \in[X]^{<\kappa_{0}}$. We shall call the tree topology on $\operatorname{Str}^{\omega}(\omega)=[\omega]^{\kappa_{0}}$ the Ellentuck topology since it is identical to the topology on $[\omega]^{\alpha_{0}}$ introduced by Ellentuck in [1].

Since we have noted that $\omega$ is just a particular $\left(\omega,<\aleph_{0}\right)$-tree, we have the following corollary to Theorem 1.9 .

Corollary 1.11 (EllentucK [1]). If $R \subseteq[\omega]^{\mathrm{N}_{0}}$ is analytic in the Ellentuck topology on $[\omega]^{\aleph_{0}}$, then $R$ is Ramsey.

Since the Ellentuck topology is finer than the classical topology, (1.11) implies Silver's partition theorem.

COROLlaRY 1.12 (SILVER [10]). If $R \subseteq[\omega]^{\pi_{0}}$ is analytic in the classical topology on $[\omega]^{\alpha_{0}}$, then $R$ is Ramsey.

2. Proof of the main theorem. In this section, we shall give a proof of Theorem 1.9. In fact, we shall prove the stronger Theorem 2.1 below.

Suppose $\tau=\langle X, \mathbf{G}\rangle$ is a topological space, i.e., $\mathbf{G}$ is the family of open subsets of the set $X$. Remember that $N \subseteq X$ is nowhere dense provided the closure of $N$ contains no nonempty open sets. A set $M \subseteq X$ is meager if it is a countable union of nowhere dense sets. And a set $B \subseteq X$ has the Baire property provided there exists an open set $U \in \mathbf{G}$ such that $B \triangle U=(B-U) \cup(U-B)$ is meager.

THEOREM 2.1. Suppose $d$ is a positive integer and $\left\langle T_{i}: i \in d\right\rangle$ is a sequence of $\left(\omega,\left\langle\aleph_{0}\right)\right.$-trees. Then a set $R \subseteq \operatorname{Str}^{\omega}\left(T_{i}: i \in d\right)$ is completely $\left\langle T_{i}: i \in d\right\rangle$-Ramsey if and only if $R$ has the Baire property in the tree topology on $\operatorname{Str}^{\omega}\left(T_{i}: i \in d\right)$.

It is well known (see Kuratowski [4, p. 94]) that each analytic set in a topology has the Baire property in that topology. Using this fact and taking $d=1$ in Theorem 2.1, we obtain Theorem 1.9. So we turn to the proof of Theorem 2.1. Our proof of (2.1) combines the ideas of Ellentuck [1], of Galvin and Prikry [3], of Nash-Williams [8] and of this author [6].

We shall need the following "pigeon-hole principle for trees" in the proof of Theorem 2.1. A proof and the history of Theorem 2.2 can be found in $\$ 2$ of [6].

Theorem 2.2 (HALPERN-LÄUChli-LAVER-Pincus). Suppose $d$ is a positive integer and $\left\langle T_{i}: i \in d\right\rangle$ is a sequence $\left(\omega,<\aleph_{0}\right)$-trees. If $F: \operatorname{Str}^{1}\left(T_{i}: i \in d\right) \rightarrow 2$ then there must exist $k \in 2$ and $\left\langle S_{i}: i \in d\right\rangle \in \operatorname{Str}^{\omega}\left(T_{i}: i \in d\right)$ such that $F$ has the constant value $k$ on $\operatorname{Str}^{1}\left(S_{i}: i \in d\right)$.

We shall also need the following strightforward lemma.

LEMMA 2.3. If $T$ is an $\left(\omega,<\aleph_{0}\right)$-tree, if $t \in T$, and if $f \in \operatorname{Incr}(\omega, \omega)$ with $f(0)=\operatorname{Lev}(t, T)$, then there must exist $S \in \operatorname{Str}_{f}(T)$ with $\operatorname{Root}(S)=t$. 
Definition 2.4. Suppose that $d$ is a positive integer and $\left\langle T_{i}: i \in d\right\rangle$ is a sequence of $\left(\omega,<\aleph_{0}\right)$-trees, and that $R \subseteq \operatorname{Str}^{\omega}\left(T_{i}: i \in d\right)$. Also, suppose that $\left\langle S_{i}\right.$ : $i \in d\rangle \in \operatorname{Str}^{\omega}\left(T_{i}: i \in d\right)$ and $\left\langle A_{i}: i \in d\right\rangle \in \operatorname{Str}^{<\omega}\left(S_{i}: i \in d\right)$. Then $\left\langle S_{i}: i \in d\right\rangle$ accepts $\left\langle A_{i}: i \in d\right\rangle$ with respect to $R$ provided $\operatorname{Str}\left(A_{i}, S_{i}: i \in d\right) \subseteq R$. We say $\left\langle S_{i}\right.$ : $i \in d\rangle$ rejects $\left\langle A_{i}: i \in d\right\rangle$ with respect to $R$ provided that each $\left\langle R_{i}: i \in d\right\rangle \in$ $\operatorname{Str}^{\omega}\left(S_{i}: i \in d\right)$ with $\left\langle A_{i}: i \in d\right\rangle \in \operatorname{Str}^{<\omega}\left(R_{i}: i \in d\right)$ does not accept $\left\langle A_{i}: i \in d\right\rangle$ with respect to $R$.

When it is clear which set $R$ is being considered, we shall omit the phrase "with respect to $R$ ".

The following lemmas build up to a proof of Theorem 2.1. In Lemmas 2.5 through 2.14 we assume that $\left\langle T_{i}: i \in d\right\rangle, R,\left\langle A_{i}: i \in d\right\rangle$ and $\left\langle S_{i}: i \in d\right\rangle$ are as described in the hypothesis of Definition 2.4.

LEMMA 2.5. If $\left\langle S_{i}: i \in d\right\rangle$ accepts (or rejects) $\left\langle A_{i}: i \in d\right\rangle$, then each

$$
\left\langle R_{i}: i \in d\right\rangle \in \operatorname{Str}^{\omega}\left(S_{i}: i \in d\right)
$$

with $\left\langle A_{i}: i \in d\right\rangle \in \operatorname{Str}^{<\omega}\left(R_{i}: i \in d\right)$ accepts (or rejects, respectively) $\left\langle A_{i}: i \in d\right\rangle$.

LEMMA 2.6. $\left\langle S_{i}: i \in d\right\rangle$ accepts (or rejects) $\left\langle A_{i}: i \in d\right\rangle$, if and only if, $\left\langle\operatorname{Dmt}\left(A_{i}, S_{i}\right): i \in d\right\rangle$ accepts (or rejects, respectively) $\left\langle A_{i}: i \in d\right\rangle$.

Lemma 2.7. There exists $\left\langle R_{i}: i \in d\right\rangle \in \operatorname{Str}\left(A_{i}, S_{i}: i \in d\right)$ such that $\left\langle R_{i}: i \in d\right\rangle$ either accepts or rejects $\left\langle A_{i}: i \in d\right\rangle$

The above lemmas are all immediate from Definition 2.4. For the next lemma, we introduce an additional definition. If $\left\langle S_{i}: i \in d\right\rangle$ either accepts or rejects $\left\langle A_{i}\right.$ : $i \in d\rangle$, then we say that $\left\langle S_{i}: i \in d\right\rangle$ decides $\left\langle A_{i}: i \in d\right\rangle$.

Lemma 2.8. Given $\left\langle T_{i}: i \in d\right\rangle$ as in Definition 2.4, there exists

$$
\left\langle R_{i}: i \in d\right\rangle \in \operatorname{Str}^{\omega}\left(T_{i}: i \in d\right)
$$

such that $\left\langle R_{i}: i \in d\right\rangle$ decides each $\left\langle B_{i}: i \in d\right\rangle \in \operatorname{Str}^{1}\left(R_{i}: i \in d\right)$.

The proof of Lemma 2.8 is not difficult. One recursively picks an array of trees $\langle T(i, n): i \in d, n \in \omega\rangle$ such that for each $i \in d$, the sequence $\langle T(i, n): n \in \omega\rangle$ decreases as a function of $n$, i.e., $T(i, n+1) \subseteq T(i, n)$. Eventually it will be that

$$
R_{i}=\bigcap_{n \in \omega} T(i, n)
$$

One can assure that the $R_{i}$ so defined are indeed $\left(\omega,<\boldsymbol{\aleph}_{0}\right)$-trees (and are strongly embedded in the $T_{i}$ ) by choosing the $T(i, n)$ with $T(i, j)(n)=T(i, n)(n)$ for all $j \geqslant n$, i.e., the $n$th level of $T(i, n)$ determines the $n$th level of all $T(i, j)$ with $j \geqslant n$, and hence the $n$th level of $R_{i}$.

Because of Lemma 2.5, we can assure that $\left\langle R_{i}: i \in d\right\rangle$ decides each $\left\langle B_{i}\right.$ : $i \in d\rangle \in \operatorname{Str}^{1}\left(R_{i}: i \in d\right)$ by selecting the $T(i, n)$ so that $\langle T(i, n): i \in d\rangle$ decides each $\left\langle B_{i}: i \in d\right\rangle \in \operatorname{Str}^{1}(T(i, n): i \in d)$ with $B_{i} \subseteq T(i, n)(n)$ for each $i$. (Then $\langle T(i, n): i \in d\rangle$ automatically decides all $\left\langle B_{i}: i \in d\right\rangle$ with $B_{i} \subseteq T(i, n)(j)$ for some $j<n$.) Such a selection of the $T(i, n)$ is easy to make using repeated applicatons of Lemma 2.7 (and of Lemma 2.3). 
Lemma 2.9. Given $\left\langle T_{i}: i \in d\right\rangle$ as assumed in Definition 2.4, there exists

$$
\left\langle R_{i}: i \in d\right\rangle \in \operatorname{Str}^{\omega}\left(T_{i}: i \in d\right)
$$

such that either $\left\langle R_{i}: i \in d\right\rangle$ accepts all $\left\langle B_{i}: i \in d\right\rangle \in \operatorname{Str}^{1}\left(R_{i}: i \in d\right)$ or $\left\langle R_{i}\right.$ : $i \in d\rangle$ rejects all $\left\langle B_{i}: i \in d\right\rangle \in \operatorname{Str}^{1}\left(R_{i}: i \in d\right)$.

The proof of Lemma 2.9 is easy. One need only apply Theorem 2.2 (HalpernLauchli-Laver-Pincus) to the result of Lemma 2.8 .

Lemma 2.10. Given $\left\langle S_{i}: i \in d\right\rangle$ as assumed in Definition 2.4, if $\left\langle S_{i}: i \in d\right\rangle$ rejects $\langle\phi: i \in d\rangle$, then there exists $\left\langle R_{i}: i \in d\right\rangle \in \operatorname{Str}^{\omega}\left(S_{i}: i \in d\right)$ such that $\left\langle R_{i}: i \in d\right\rangle$ rejects all $\left\langle B_{i}: i \in d\right\rangle \in \operatorname{Str}^{1}\left(R_{i}: i \in d\right)$.

The $\left\langle R_{i}: i \in d\right\rangle$ from Lemma 2.9 must satisfy Lemma 2.10; otherwise Lemma 2.9 yields that $\left\langle R_{i}: i \in d\right\rangle$ accepts all $\left\langle B_{i}: i \in d\right\rangle \in \operatorname{Str}^{1}\left(R_{i}: i \in d\right)$. Then $\operatorname{Str}^{\omega}\left(R_{i}: i \in d\right) \subseteq R$, and $\left\langle S_{i}: i \in d\right\rangle$ would not reject $\langle\phi: i \in d\rangle$.

Lemma 2.11. Given $\left\langle S_{i}: i \in d\right\rangle$ and $\left\langle A_{i}: i \in d\right\rangle$ as in the supposition of Definition 2.4, let $N=\operatorname{Height}\left(A_{i}\right)$. If $\left\langle S_{i}: i \in d\right\rangle$ rejects $\left\langle A_{i}: i \in d\right\rangle$, then there exists

$$
\left\langle R_{i}: i \in d\right\rangle \in \operatorname{Str}\left(A_{i}, S_{i}: i \in d\right)
$$

such that $\left\langle R_{i}: i \in d\right\rangle$ rejects all $\left\langle B_{i}: i \in d\right\rangle \in \operatorname{Str}^{N+1}\left(R_{i}: i \in d\right)$ with $A_{i}<{ }^{*} B_{i}$ for each $i \in d$.

If $\left\langle S_{i}: i \in d\right\rangle$ and $\left\langle A_{i}: i \in d\right\rangle$ satisfy the hypothesis of Lemma 2.11 , then we can assume $A_{i}<^{*} S_{i}$ for each $i \in d$. Letting $N=\operatorname{Height}\left(A_{i}\right)$, we can write each $S_{i}-A_{i}$ as a union of disjoint sets

$$
S_{i}-A_{i}=\cup\left\{\operatorname{Succ}\left(a, S_{i}\right): \text { there exists } b \in A_{i}(N-1) \text { with } a \in \operatorname{IS}\left(b, S_{i}\right)\right\} .
$$

We shall concentrate on the array of trees

$$
\left\langle\operatorname{Succ}\left(a, S_{i}\right): i \in d \text { and there exists } b \in A_{i}(N-1) \text { with } a \in \operatorname{IS}\left(b, S_{i}\right)\right\rangle \text {. }
$$

(We consider $\operatorname{Succ}\left(a, S_{i}\right)$ a tree by giving it the induced partial order.) Since (1) is cumbersome to write, we shall make the notational convention that $M_{i}=$ $\cup_{b \in A_{i}(N-1)} \operatorname{IS}\left(b, S_{i}\right)$, so (1) becomes

$$
\left\langle\operatorname{Succ}\left(a, S_{i}\right): i \in d, a \in M_{i}\right\rangle .
$$

We define

$$
R^{\prime} \subseteq \operatorname{Str}^{\omega}\left(\operatorname{Succ}\left(a, S_{i}\right): i \in d, a \in M_{i}\right)
$$

by $\left\langle Q(a, i): i \in d, a \in M_{i}\right\rangle \in R^{\prime}$ if and only if $\left\langle Q(a, i): i \in d, a \in M_{i}\right\rangle \in$ $\operatorname{Str}^{\omega}\left(\operatorname{Succ}\left(a, S_{i}\right): i \in d, a \in M_{i}\right)$ and $\left\langle\left(\cup_{a \in M_{i}} Q(a, i)\right) \cup A_{i}: i \in d\right\rangle \in R$. Then to prove Lemma 2.11 one applies Lemma 2.10 to the sequence of trees (2) and the set $R^{\prime}$.

Lemma 2.12. Given $\left\langle S_{i}: i \in d\right\rangle$ and $\left\langle A_{i}: i \in d\right\rangle$ as assumed in Definition 2.4, suppose $\left\langle S_{i}: i \in d\right\rangle$ rejects $\left\langle C_{i}: i \in d\right\rangle, N$ is a positive integer, $\operatorname{Height}\left(C_{i}\right)=N$,

$$
\left\langle C_{i}: i \in d\right\rangle \in \operatorname{Str}^{N}\left(A_{i}: i \in d\right),
$$


and every maximal node of $C_{i}$ is also maximal in the corresponding $A_{i}$. Then there must exist $\left\langle R_{i}: i \in d\right\rangle \in \operatorname{Str}\left(A_{i}, S_{i}: i \in d\right)$ which rejects all $\left\langle B_{i}: i \in d\right\rangle \in$ $\operatorname{Str}^{N+1}\left(R_{i}: i \in d\right)$ with $C_{i}<^{*} B_{i}$ for each $i \in d$.

Lemma 2.12 is a straightforward generalization of Lemma 2.11. Using a recursive definition similar to the one in the proof of Lemma 2.8 along with repeated applications of Lemma 2.12, one can prove the following lemma.

Lemma 2.13. Given $\left\langle S_{i}: i \in d\right\rangle$ as in Definition 2.4, if $\left\langle S_{i}: i \in d\right\rangle$ rejects $\langle\phi$ : $i \in d\rangle$, then there exists $\left\langle R_{i}: i \in d\right\rangle \in \operatorname{Str}^{\omega}\left(S_{i}: i \in d\right)$ such that $\left\langle R_{i}: i \in d\right\rangle$ rejects all $\left\langle B_{i}: i \in d\right\rangle \in \operatorname{Str}^{<\omega}\left(R_{i}: i \in d\right)$.

Also, just as Lemma 2.10 was generalized to Lemma 2.11, so from Lemma 2.13 we obtain the following lemma.

Lemma 2.14. Given $\left\langle S_{i}: i \in d\right\rangle$ and $\left\langle A_{i}: i \in d\right\rangle$ as in Definition 2.4, if $\left\langle S_{i}\right.$ : $i \in d\rangle$ rejects $\left\langle A_{i}: i \in d\right\rangle$, then there exists

$$
\left\langle R_{i}: i \in d\right\rangle \in \operatorname{Str}\left(A_{i}, S_{i}: i \in d\right)
$$

such that $\left\langle R_{i}: i \in d\right\rangle$ rejects all $\left\langle C_{i}: i \in d\right\rangle \in \operatorname{Str}^{<\omega}\left(R_{i}: i \in d\right)$ with $A_{i}<{ }^{*} C_{i}$ for each $i \in d$.

We shall present more detailed proofs of the following lemmas.

LEMMA 2.15. Suppose $d$ is a positive integer, $\left\langle T_{i}: i \in d\right\rangle$ is a sequence of $\left(\omega,<\aleph_{0}\right)$-trees, and that $R \subseteq \operatorname{Str}^{\omega}\left(T_{i}: i \in d\right)$ is an open set in the tree topology on $\operatorname{Str}^{\omega}\left(T_{i}: i \in d\right)$. Then $R$ is completely $\left\langle T_{i}: i \in d\right\rangle$-Ramsey.

Proof. Suppose that $R$ and $\left\langle T_{i}: i \in d\right\rangle$ satisfy the hypothesis. Also, suppose $\left\langle S_{i}: i \in d\right\rangle \in \operatorname{Str}^{\omega}\left(T_{i}: i \in d\right)$ and $\left\langle A_{i}: i \in d\right\rangle \in \operatorname{Str}^{<\omega}\left(S_{i}: i \in d\right)$.

If some $\left\langle R_{i}: i \in d\right\rangle \in \operatorname{Str}\left(A_{i}, S_{i}: i \in d\right)$ accepts $\left\langle A_{i}: i \in d\right\rangle$, then

$$
\operatorname{Str}\left(A_{i}, R_{i}: i \in d\right) \subseteq R,
$$

and we are done.

Otherwise $\left\langle S_{i}: i \in d\right\rangle$ rejects $\left\langle A_{i}: i \in d\right\rangle$. So apply Lemma 2.14 to obtain $\left\langle R_{i}\right.$ : $i \in d\rangle \in \operatorname{Str}\left(A_{i}, S_{i}: i \in d\right)$ such that $\left\langle R_{i}: i \in d\right\rangle$ rejects each $\left\langle C_{i}: i \in d\right\rangle \in$ $\operatorname{Str}^{<\omega}\left(R_{i}: i \in d\right)$ with $A_{i}<^{*} C_{i}$ for each $i \in d$. We claim $\operatorname{Str}\left(A_{i}, R_{i}: i \in d\right) \cap R=$ $\varnothing$.

Suppose not and pick $\left\langle Q_{i}: i \in d\right\rangle \in \operatorname{Str}\left(A_{i}, R_{i}: i \in d\right) \cap R$. Since

$$
\operatorname{Str}\left(A_{i}, R_{i}: i \in d\right) \cap R
$$

is open, we can find a basic open $\operatorname{set} \operatorname{Str}\left(B_{i}, P_{i}: i \in d\right)$ with

$$
\left\langle Q_{i}: i \in d\right\rangle \in \operatorname{Str}\left(B_{i}, P_{i}: i \in d\right) \subseteq \operatorname{Str}\left(A_{i}, R_{i}: i \in d\right) \cap R .
$$

In fact, we can assume $A_{i}<{ }^{*} B_{i}<{ }^{*} P_{i}$, for each $i \in d$, and $\left\langle P_{i}\right.$ : $\left.i \in d\right\rangle \in$ $\operatorname{Str}\left(A_{i}, R_{i}: i \in d\right)$. Then $\left\langle P_{i}: i \in d\right\rangle$ accepts $\left\langle B_{i}: i \in d\right\rangle$, but this contradicts the requirement that $\left\langle R_{i}: i \in d\right\rangle$ rejects $\left\langle B_{i}: i \in d\right\rangle$. The contradiction proves the lemma. 
Lemma 2.16. Suppose $\left\langle T_{i}: i \in d\right\rangle$ is a finite sequence of $\left(\omega,<\aleph_{0}\right)$-trees, and $N \subseteq \operatorname{Str}^{\omega}\left(T_{i}: i \in d\right)$ is nowhere dense in the tree topology. Then for each $\left\langle S_{i}\right.$ : $i \in d\rangle \in \operatorname{Str}^{\omega}\left(T_{i}: i \in d\right)$ and each $\left\langle A_{i}: i \in d\right\rangle \in \operatorname{Str}^{<\omega}\left(S_{i}: i \in d\right)$, there must exist $\left\langle R_{i}: i \in d\right\rangle \in \operatorname{Str}\left(A_{i}, S_{i}: i \in d\right)$ with $\operatorname{Str}\left(A_{i}, R_{i}: i \in d\right) \cap N=\varnothing$.

This is immediate from Lemma 2.15 applied to the complement of the closure of $N$.

LemMA 2.17. Suppose $\left\langle T_{i}: i \in d\right\rangle$ is a finite sequence of $\left(\omega,<\kappa_{0}\right)$-trees; then $M \subseteq \operatorname{Str}^{\omega}\left(T_{i}: i \in d\right)$ is meager in the tree topology if and only if $M$ is nowhere dense in the tree topology.

Proof. If $M \subseteq \operatorname{Str}^{\omega}\left(T_{i}: i \in d\right)$ is nowhere dense, then $M$ is trivially meager.

So suppose $M=\cup_{n \in \omega} N_{n}$ where each $N_{n} \subseteq \operatorname{Str}^{\omega}\left(T_{i}: i \in d\right)$ is nowhere dense. In order to conclude that $M$ is nowhere dense, it suffices to show that for each nonempty, open $R \subseteq \operatorname{Str}^{\omega}\left(T_{i}: i \in d\right)$, there exists a basic open neighborhood $\operatorname{Str}\left(A_{i}, R_{i}: i \in d\right)$ with $\operatorname{Str}\left(A_{i}, R_{i}: i \in d\right) \subseteq R-M$.

So assume such $R$ is given, and pick $\left\langle S_{i}: i \in d\right\rangle \in \operatorname{Str}^{\omega}\left(T_{i}: i \in d\right)$ and $\left\langle A_{i}\right.$ : $i \in d\rangle \in \operatorname{Str}^{<\omega}\left(S_{i}: i \in d\right)$ so that $\operatorname{Str}\left(A_{i}, S_{i}: i \in d\right) \subseteq R$. Let $\operatorname{Height}\left(A_{i}\right)=H$, for each $i \in d$.

By induction on $n, n \in \omega$, we shall define two arrays of trees, $\langle T(i, n): i \in d$, $n \in \omega\rangle$ and $\langle P(i, n): i \in d, n \in \omega\rangle$, such that the following conditions hold for each $n \in \omega$.

(a) $\langle T(i, n): i \in d\rangle \in \operatorname{Str}\left(A_{i}, S_{i}: i \in d\right)$.

(b) $\langle P(i, 0): i \in d\rangle=\left\langle A_{i}: i \in d\right\rangle$, and if $n>1$, then for each $i \in d, P(i, n)=$ $\cup_{k \in n+H} T(i, n-1)(k)$, and $P(i, n)$ has the induced partial order.

(c) If $n \geqslant 1$, then

$$
\langle T(i, n): i \in d\rangle \in \operatorname{Str}(P(i, n), T(i, n-1): i \in d) .
$$

(d) Suppose $H \leqslant k \leqslant n+H$ and $\left\langle B_{i}: i \in d\right\rangle \in \operatorname{Str}^{k}(P(i, n): i \in d)$ with $A_{i}$ $\left\langle{ }^{*} B_{i}\right.$ for each $i \in d$. Then for every $\left\langle Q_{i}: i \in d\right\rangle \in \operatorname{Str}\left(B_{i}, T(i, n): i \in d\right)$ with $Q_{i} \cap P(i, n)=B_{i}$ for each $i \in d$, we have $\left\langle Q_{i}: i \in d\right\rangle \notin N_{n}$.

If $n=0$, then condition (b) defines $\langle P(i, 0): i \in d\rangle=\left\langle A_{i}: i \in d\right\rangle$. So we can apply Lemma 2.16 to get $\langle T(i, 0): i \in d\rangle \in \operatorname{Str}\left(A_{i}, S_{i}: i \in d\right)$ such that $\operatorname{Str}\left(A_{i}, T(i, 0): i \in d\right) \cap N_{0}=\varnothing$.

Given $n \geqslant 1$ and the trees $T(i, k)$ and $P(i, k)$ for each $i \in d$ and $k \in n$, we want to select $T(i, n)$ and $P(i, n)$ for each $i \in d$. Now condition (b) determines $\langle P(i, n)$ : $i \in d\rangle$ and hence $T(i, n)(j)$ for each $j \in n+H$ because of condition (c). So it remains to select $T(i, n)(j)$ for $j>n+H$.

Let $P^{\prime}(i, n)=\cup_{k \in n+H+1} T(i, n-1)(k)$ for each $i \in d$, and let

$$
\begin{aligned}
\mathcal{C}(n)=\{\langle C(i): i & \in d\rangle \in \operatorname{Str}^{<n+H+1}\left(P^{\prime}(i, n): i \in d\right): \text { for each } i \in d, \\
A_{i} & \left.<{ }^{*} C(i) \text { and } C(i)(\operatorname{Height}(C(i)-1)) \subseteq P^{\prime}(i, n)(n+H)\right\} .
\end{aligned}
$$

Let $K=|\Theta(n)|$ and enumerate $\varrho(n)$ as $\{\mathbf{C}(p): 1<p<K\}$ where $\mathbf{C}(p)=$ $\langle C(p, i): i \in d\rangle$. 
By induction on $p, p \in K+1$, we shall define trees $T(i, n, p)$ such that the following conditions hold for each $p \in K+1$.

(e) $T(i, n, 0)=T(i, n-1)$ for each $i \in d$.

(f) If $p \geqslant 1$, then $\langle T(i, n, p): i \in d\rangle \in \operatorname{Str}\langle(P(i, n), T(i, n, p-1): i \in d)\rangle$.

(g) Write $B_{i}=C(p, i) \cap P(i, n)$, and $H(p)=\operatorname{Height}(C(p, i))$, and $I(i)=$ $C(p, i)(H(p)-1)$ for each $i \in d$.

If

$$
V(i)=B_{i} \cup\left(\bigcup\left\{\operatorname{Succ}\left(a, S_{i}\right) \cap T(i, n, p): a \in I(i)\right\}\right)
$$

has the induced partial order, then

$$
\operatorname{Str}\left(B_{i}, V(i): i \in d\right) \cap N_{n}=\varnothing \text {. }
$$

Condition (e) defines $T(i, n, 0)$. So suppose $p>1$ and the trees $T(i, n, q)$ have been defined for $i \in d$ and $q \in p$. We shall use the notational conventions made in the first sentence of conditions $(\mathrm{g})$. Let

$$
U(i)=B_{i} \cup\left(\bigcup\left\{\operatorname{Succ}\left(a, S_{i}\right) \cap T(i, n, p-1): a \in I(i)\right\}\right) .
$$

Then apply Lemma 2.16 to $\langle U(i): i \in d\rangle$ and obtain $\langle V(i): i \in d\rangle \in$ $\operatorname{Str}\left(B_{i}, U(i): i \in d\right)$ so that

$$
\operatorname{Str}\left(B_{i}, V(i): i \in d\right) \cap N_{n}=\varnothing .
$$

But then we can use Lemma 2.3 to find

$$
\langle T(i, n, p): i \in d\rangle \in \operatorname{Str}(P(i, n), T(i, n, p-1): i \in d)
$$

such that for each $i \in d$ and each $a \in I(i)$,

$$
\operatorname{Succ}\left(a, S_{i}\right) \cap T(i, n, p)=\operatorname{Succ}\left(a, S_{i}\right) \cap V(i) .
$$

This assures that equation (1) holds, so the conditions (f) and (g) hold.

When the induction on $p \in K+1$ is complete, we set $T(i, n)=T(i, n, K)$, so the conditions (a) - (c) follow immediately. And condition (d) follows from condition (g) after a moment of thought. So we have completed our induction on $n \in \omega$.

By conditions (a)-(c) we can set

$$
R_{i}=\bigcap_{n \in \omega}(T(i, n))=A_{i} \cup\left(\bigcup_{n \in \omega} T(i, n)(n+H-1)\right)=\bigcup_{n \in \omega} P(i, n)
$$

for each $i \in d$, and get $\left\langle R_{i}: i \in d\right\rangle \in \operatorname{Str}\left(A_{i}, S_{i}: i \in d\right)$.

Now, it is clear that $\operatorname{Str}\left(A_{i}, R: i \in d\right) \subseteq \operatorname{Str}\left(A_{i}, S_{i}: i \in d\right) \subseteq R$, and we claim $\operatorname{Str}\left(A_{i}, R_{i}: i \in d\right) \cap M=\varnothing$ (which, if true, proves the lemma). Indeed, suppose $\left\langle Q_{i}: i \in d\right\rangle \in \operatorname{Str}\left(A_{i}, R_{i}: i \in d\right) \cap N_{n}$ for some $n \in \omega$. Let $B_{i}=Q_{i} \cap P(i, n)$ for each $i \in d$. Then $\left\langle Q_{i}: i \in d\right\rangle$ and $\left\langle B_{i}: i \in d\right\rangle$ satisfy the hypothesis of condition (d), and we conclude $\left\langle Q_{i}: i \in d\right\rangle \notin N_{n}$. This contradiction proves the lemma.

Lemmas 2.16-2.17 enable us to prove Theorem 2.1.

Proof of Theorem 2.1. Suppose $\left\langle T_{i}: i \in d\right\rangle$ is a finite sequence of $\left(\omega,\left\langle\aleph_{0}\right)\right.$ trees. 
If $R \subseteq \operatorname{Str}^{\omega}\left(T_{i}: i \in d\right)$ has the Baire property (i.e., $R \triangle U=(R-U) \cup(U-$ $R$ ) is meager for some open set $U$ ), then we want to show $R$ is completely $\left\langle T_{i}\right.$ : $i \in d\rangle$-Ramsey. Now Lemma 2.17 states that $R \triangle U$ is in fact nowhere dense (in the tree topology). So suppose $\left\langle S_{i}: i \in d\right\rangle \in \operatorname{Str}^{\omega}\left(T_{i}: i \in d\right)$ and $\left\langle A_{i}: i \in d\right\rangle \in$ $\operatorname{Str}^{<\omega}\left(T_{i}: i \in d\right)$. Since $U$ is open, Lemma 2.15 implies there exists $\left\langle R_{i}: i \in d\right\rangle \in$ $\operatorname{Str}\left(A_{i}, S_{i}: i \in d\right)$ with either $\operatorname{Str}\left(A_{i}, R_{i}: i \in d\right) \subseteq U$ or $\operatorname{Str}\left(A_{i}, R_{i}: i \in d\right) \cap U=$ $\varnothing$. But the fact that $R \triangle U$ is nowhere dense and Lemma 2.16 yield $\left\langle Q_{i}: i \in d\right\rangle \in$ $\operatorname{Str}\left(A_{i}, R_{i}: i \in d\right)$ such that

$$
\operatorname{Str}\left(A_{i}, Q_{i}: i \in d\right) \cap(R \triangle U)=\varnothing .
$$

Thus $\operatorname{Str}\left(A_{i}, R_{i}: i \in d\right) \subseteq \cup$ implies $\operatorname{Str}\left(A_{i}, Q_{i}: i \in d\right) \subseteq R$, while

$$
\operatorname{Str}\left(A_{i}, R_{i}: i \in d\right) \cap U=\varnothing
$$

implies $\operatorname{Str}\left(A_{i}, Q_{i}: i \in d\right) \cap R=\varnothing$.

Conversely, suppose $R$ is completely $\left\langle T_{i}: i \in d\right\rangle$-Ramsey. Let $\operatorname{int}(R)$ be the interior of $R$. We shall show that $R-\operatorname{int}(R)$ is nowhere dense. To show this, it suffices to show that for each nonempty, open set $U$, there exists a basic open set $\operatorname{Str}\left(A_{i}, R_{i}: i \in d\right) \subseteq U-(R-\operatorname{int}(R))$.

Indeed, given nonempty open $U$, pick $\left\langle S_{i}: i \in d\right\rangle \in \operatorname{Str}^{\omega}\left(T_{i}: i \in d\right)$ and $\left\langle A_{i}\right.$ : $i \in d\rangle \in \operatorname{Str}^{<\omega}\left(S_{i}: i \in d\right)$ such that $\operatorname{Str}\left(A_{i}, S_{i}: i \in d\right) \subseteq U$. Since $R$ is completely $\left\langle T_{i}: i \in d\right\rangle$-Ramsey, there must exist $\left\langle R_{i}: i \in d\right\rangle \in \operatorname{Str}\left(A_{i}, S_{i}: i \in d\right)$ with either $\operatorname{Str}\left(A_{i}, R_{i}: i \in d\right) \subseteq R$ or $\operatorname{Str}\left(A_{i}, R_{i}: i \in d\right) \cap R=\varnothing$. In the first case, $\operatorname{Str}\left(A_{i}, R_{i}: i \in d\right)$ is open, so $\operatorname{Str}\left(A_{i}, R_{i}: i \in d\right) \subseteq \operatorname{int}(R)$. So in either case, $\operatorname{Str}\left(A_{i}, R_{i}: i \in d\right) \subseteq U-(R-\operatorname{int}(R))$. This complete the proof of Theorem 2.1.

3. A Nash-Williams partition theorem for trees. A family of finite sets $\mathscr{Q} \subseteq[\omega]^{<\kappa_{0}}$ is said to be thin provided it is not the case that there exist distinct sets $A, B \in \mathbb{Q}$ with $A \ll B$. In [8], Nash-Williams proved the following generalization of Ramsey's theorem.

TheOREM 3.1 (NASH-WILliams). Suppose that $\mathbb{Q} \subseteq[\omega]^{<\kappa_{0}}$ is thin, that $r$ is a positive integer, and that $\mathbb{Q}=\cup_{i \in r} C_{i}$. Then there must exist $X \in[\omega]^{\alpha_{0}}$ and $k \in r$ such that $\mathbb{Q} \cap[X]^{<*_{0}} \subseteq C_{k}$.

We shall show that Theorem 1.9 implies a generalization for trees of Theorem 3.1 .

Definition 3.2. Suppose that $T$ is an $\omega$-tree. A family of subtrees $\mathscr{B} \subseteq \operatorname{Str}^{<\omega}(T)$ is said to be thin provided that it is not the case that there exist distinct trees $A$, $B \in \mathscr{B}$ with $A<{ }^{*} B$.

Theorem 3.3. Suppose that $T$ is an $\left(\omega,<\aleph_{0}\right)$-tree, that $\mathscr{B} \subseteq \mathrm{Str}^{<\omega}(T)$ is thin, that $r$ is a positive integer, and that $\mathscr{B}=\cup_{i \in r} C_{i}$. Then there must exist $S \in \operatorname{Str}^{\omega}(T)$ and $k \in r$ such that $\mathscr{B} \cap \operatorname{Str}^{<\omega}(S) \subseteq C_{k}$.

Theorem 3.3 becomes Theorem 3.1 if we take $T$ to be the trivial $(\omega, 1)$-tree, i.e., $T=\omega$. Also, note that for each $n \in \omega$, it is clear that $\operatorname{Str}^{n}(T)$ is a thin family of subtrees whenever $T$ is an $\omega$-tree. Hence, we have the following generalization for trees of Ramsey's theorem. 
COROllary 3.4. Suppose that $T$ is an $\left(\omega,<\aleph_{0}\right)$-tree, that $n$ and $r$ are positive integers, and that $\operatorname{Str}^{n}(T) \subseteq \cup_{i \in r} C_{i}$. Then there must exist $k \in r$ and $S \in \operatorname{Str}^{\omega}(T)$ with $\operatorname{Str}^{n}(S) \subseteq C_{k}$.

A finitary version of (3.4) and related results can be found in [6].

Proof of Theorem 3.3. Suppose that $T$ and $\mathscr{B}$ satisfy the hypothesis. By a standard argument, we may assume that $r=2$. So suppose $\mathscr{B}=C_{0} \cup C_{1}$. Define

$$
P=\left\{R \in \operatorname{Str}^{\omega}(T): \text { there exists } A \in C_{0} \text { with } A<{ }^{*} R\right\} .
$$

Since $C_{0} \subseteq \operatorname{Str}^{<\omega}(T)$, it must be that $P$ is an open set in the tree topology on $\operatorname{Str}^{\omega}(T)$. Thus Theorem 1.9 (or Lemma 2.15) implies that there exists $S \in \operatorname{Str}^{\omega}(T)$ with either $\operatorname{Str}^{\omega}(S) \subseteq P$ or $\operatorname{Str}^{\omega}(S) \cap P=\varnothing$.

If $\operatorname{Str}^{\omega}(S) \subseteq P$, then the fact that $\mathscr{B}$ is thin requires $\mathscr{B} \cap \operatorname{Str}^{<\omega}(S) \subseteq C_{0}$. Similarly, if $\operatorname{Str}^{\omega}(S) \cap P=\varnothing$, then $C_{0} \cap \operatorname{Str}^{<\omega}(S)=\varnothing$, so $\mathscr{B} \cap \operatorname{Str}^{<\omega}(S) \subseteq C_{1}$. This proves Theorem 3.3.

\section{REFERENCES}

1. E. Ellentuck, $A$ new proof that analytic sets are Ramsey, J. Symbolic Logic 39 (1974), 163-165.

2. P. Erdös and R. Rado, Combinatorial theorems on classifications of subsets of a given set, Proc. London Math. Soc. (3) 2 (1952), 417-439.

3. F. Galvin and K. Prikry, Borel sets and Ramsey's theorem, J. Symbolic Logic 38 (1973), 193-198.

4. C. Kuratowski, Topology, Vol. 1 (English rev. ed.), Academic Press, New York, 1966.

5. A. Louveau, Demonstration topologique de théorèmes de Silver et Mathias, Bull. Sci. Math. (2) 98 (1974), 97-102.

6. K. R. Milliken, A Ramsey theorem for trees, J. Combin. Theory Ser. A 26 (1979), 215-237.

7. Some results in Ramsey theory, Doctoral Dissertation, U.C.L.A., 1975.

8. C. St. J. A. Nash-Williams, On well-quasi-ordering transfinite sequences, Proc. Cambridge Philos. Soc. 61 (1965), 33-39.

9. F. P. Ramsey, On a problem of formal logic, Proc. London Math. Soc. (2) 30 (1929), 264-286.

10. J. Silver, Every analytic set is Ramsey, J. Symbolic Logic 35 (1970), 60-64.

11. A Taylor, Some results in partition theory, Doctoral Dissertation, Dartmouth College, 1975.

Department of Computer Science, ibM Thomas J. Watson Research Center, Yorktown HeIGHTS, NeW YORK 10598 